\title{
PAPER \\ Dynamic Performance Adjustment of CPU and GPU in a Gaming Notebook at the Battery Mode
}

\section{Chun-Hung CHENG ${ }^{\dagger, \dagger+a)}$, Student Member and Ying-Wen $\mathrm{BAI}^{\dagger}$, Nonmember}

SUMMARY This new design uses a low power embedded controller (EC) in cooperation with the BIOS of a notebook (NB) computer, both to accomplish dynamic adjustment and to maintain a required performance level of the battery mode of the notebook. In order to extend the operation time at the battery mode, in general, the notebook computer will directly reduce the clock rate and then reduce the performance. This design can obtain the necessary balance of the performance and the power consumption by using both the EC and the BIOS cooperatively to implement the dynamic control of both the CPU and the GPU frequency to maintain the system performance at a sufficient level for a high speed and high resolution video game. In contrast, in order to maintain a certain notebook performance, in terms of battery life it will be necessary to make some trade-offs.

key words: gaming notebook, high resolution video game, embedded controller, battery discharge

\section{Introduction}

In recent years, with the rise of the gaming notebook, computer developers focusing on business-oriented notebooks have paid special attention to the design of the gaming notebook. In particular, a gaming notebook is easy to carry, has a higher performance, significantly lessens the difference between common notebook computers and desktop computers, and has become the new darling of computer-related products. Figure 1 shows the total annual notebook computer shipments worldwide has been declining year by year. However, according to Fig. 2, the percentage of the annual shipments of gaming notebooks is increasing year by year. The market sharing shows that gaming notebooks have gradually become the user's favorite [23], [24].

Faced with the low-cost Tablet PC competition, the common notebook portion of the global notebook market is gradually declining. The processor manufacturers have had a certain direct impact on the common notebook computer processor. As with the common notebook market recession, since the consumer market is mainly the result of the market sharing of the Tablet PC, in order to restore market demand PC manufacturers have turned their product design to gaming notebooks.

As the gaming notebook price is higher, but the market size is limited, and as the brand long-term business can dominate the market sharing, in the past, there were only

Manuscript received July 6, 2018.

Manuscript revised January 23, 2019.

Manuscript publicized March 27, 2019.

${ }^{\dagger}$ The authors are with Fu Jen Catholic University, 24205 Taiwan, R.O.C.

${ }^{\dagger \dagger}$ The author is also with CLEVO Company, Taiwan, R.O.C.

a)E-mail: 400068058@mail.fju.edu.tw

DOI: 10.1587/transinf.2018EDP7241

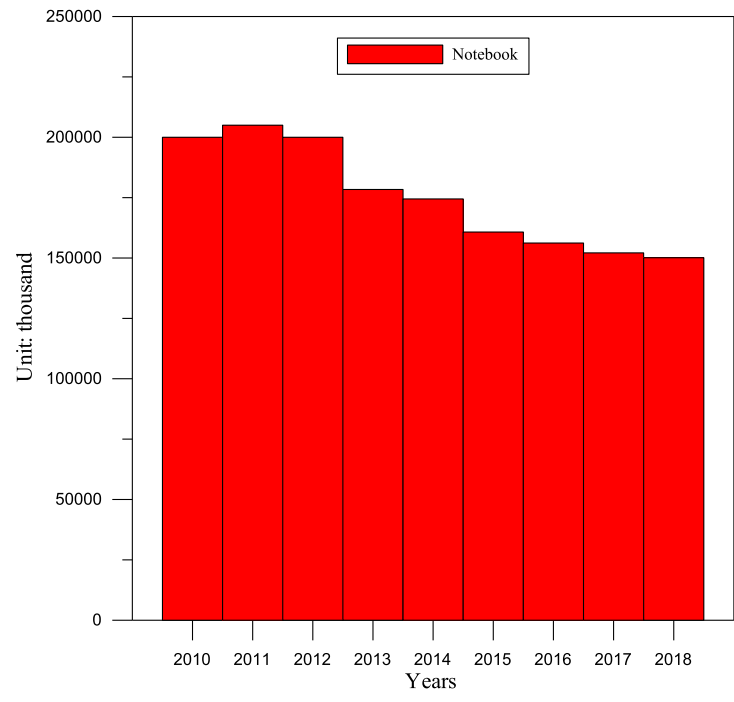

Fig. $1 \quad 2010-2018$ global notebook market.

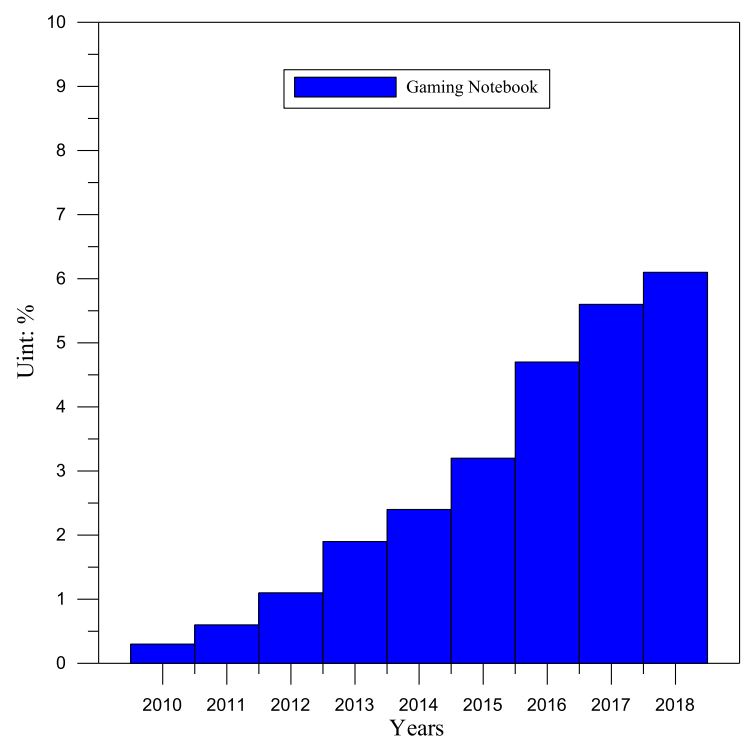

Fig. 2 2010-2018 gaming notebook percentage share of the global notebook market.

a few brands that had a long-term period of business. But when the notebook market changed, the low-cost gaming notebook development, the competitive consumer market demands for electricity stability, and the high gross profit margins, encouraged brand manufacturers to develop their 
Table 1 A comparison of a common notebook, a low end gaming notebook, a high end gaming notebook, and our gaming notebook.

\begin{tabular}{ccccc}
\hline & $\begin{array}{c}\text { Common } \\
\text { Notebook }\end{array}$ & $\begin{array}{c}\text { Low end } \\
\text { Gaming NB }\end{array}$ & $\begin{array}{c}\text { High end } \\
\text { Gaming } \\
\text { NB }\end{array}$ & $\begin{array}{c}\text { Our Gaming } \\
\text { NB }\end{array}$ \\
\hline $\begin{array}{c}\text { Performance of AC } \\
\text { mode }\end{array}$ & Low & High & High & High \\
\hline $\begin{array}{c}\text { Performance of } \\
\text { battery mode }\end{array}$ & Low & Low & High & High \\
\hline Power consumption & Low & $\begin{array}{c}\text { High } \\
(220 \mathrm{~W}-\end{array}$ & $\begin{array}{c}\text { High } \\
(330 \mathrm{~W}-)\end{array}$ & $\begin{array}{c}\text { High } \\
(220 \mathrm{~W}- \\
330 \mathrm{~W})\end{array}$ \\
\hline Mobility & Yes & No & No & No \\
\hline Cost & Low & High & Higher & High \\
\hline
\end{tabular}

gaming notebooks further [1].

Table 1 shows a specification comparison table of a common notebook, a low end gaming notebook, a high end gaming notebook, and our gaming notebook. The common notebook computer focuses on mobility, but as the gaming notebook focuses on both high-performance and high resolution display, the relative cost is higher.

As the common notebook market has gradually entered the mature stage, the gaming notebook market has gradually become a new sub-line. The video game, a cost-effective nature of the application, is the usual target because of its power consumption. Thus a higher performance is the major focus. A high performance is the most important feature of gaming notebooks, but in order to extend the battery life while in the battery mode, the designer will directly reduce the performance in order to reduce power consumption, so as to maintain a high-performance state, which can be a dilemma in the battery mode [2]-[5]. Other major laptop designers emphasize the performance that is not focused on the battery mode. Usually, the battery mode is also the design of the common notebook by reducing its performance to extend the battery life. Hence, our design is for the battery mode gaming notebook which can have both a higher performance and a longer battery life by balancing the clock rate both of the CPU and of the GPU [6]-[9]. The design, therefore, sacrifices some battery life exchange for a higher performance state [10], [11].

Other related design is the use of the Dynamic Voltage Frequency Scaling (DVFS) method to provide the dynamic adjustment CPU-GPU DVFS. Our design uses a simple detection of the discharge " $\mathrm{C}$ " number to achieve the CPUGPU balance control. Our design need not add a complicated hardware circuit; instead, our design only use the EC and the BIOS cooperation to achieve the CPU-GPU balance control [20].

This design is for a high end notebook which has a $4 \mathrm{~K}$ resolution display or QFHD $(3840 \times 2160)$. A current gaming notebook with this design can provide the required 30 FPS or more for a high resolution video game experience. According to recent statistics, as a typical video game frame speed is maintained at around 30 FPS, the user will not feel the display lag phenomenon [12], [13]. This design will insure a constant frame speed of 30 FPS. In addition, this design is a fully automated technological device. As long as the game notebook is not connected to the power supply when the user plays video games, this mode will start immediately.

The design requirement allows users to enjoy an excellent system's performance quality either in the AC mode or in the battery mode. Hence a user plays a game on a gaming notebook whose performance cannot be limited because of the battery model. In the AC mode, as this design need not test the battery condition, the system performance can be increased to maintain the performance of more than 30 FPS. Usually, the previous gaming notebook system performance will be reduced by more than $50 \%$ during the battery mode operation. Therefore this design adds an embedded processor and BIOS cooperation mechanism to dynamically enhance the system's performance when the gaming notebook is operated in the battery mode.

\section{Hardware and Software Design of a Gaming Note- book}

The common notebook design focuses on three key areas: performance, power consumption, and thermal. When the common notebook is in the battery mode, in order to extend the battery life, the design will reduce the CPU and GPU clock frequency, so that the system operates at a low power state. This is because when the common notebook is considered in terms of mobility, as its battery life is long, the common notebook, therefore, can sacrifice the performance in exchange for a longer battery life.

The gaming notebook design considerations focus on performance and thermal, so the design considerations may sacrifice the battery life in exchange for better performance. This design, which is for high-performance notebooks, when in the battery mode, can also maintain the required video game frame quality.

Our design uses a low power embedded controller (EC) for this gaming notebook as shown in Fig. 3. The EC connects both to the south bridge, and to the BIOS, and controls the external devices [14]-[18].

As shown in Fig. 4, the EC will immediately detect the discharge " $C$ " number of the battery, and then according to the level of the number of the discharge "C" number will inform the BIOS to control the clock rates of the CPU and the GPU to either increase or reduce the clock speed. In this design, the EC communicates with the BIOS via the low pin count bus (LPC) IO Port: 0x62/0x66.

As the "Embedded Controller" and the "BIOS" in Fig. 4 are all firmware in the notebook architecture, we will put the "Embedded Controller" and "BIOS" in the Firmware design in Fig. 4.

Figure 3 is the architecture diagram of the entire notebook, which contains the CPU, PCH, BIOS, EC and other devices. Figure 4 shows the structure of the key part of this design, which is part of Fig. 3. The bi-directional arrow indicates the action that the EC/BIOS can read or write directly. The single-end arrow indicates that the EC/BIOS can only perform write operations. 


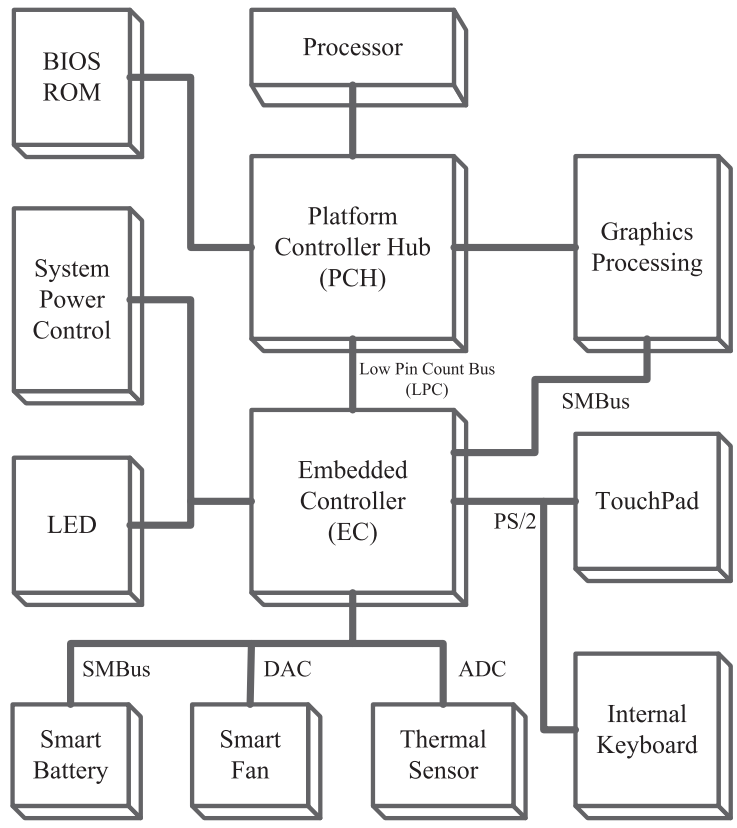

Fig. 3 The system architecture of the notebook and the EC.

\section{Firmware design}

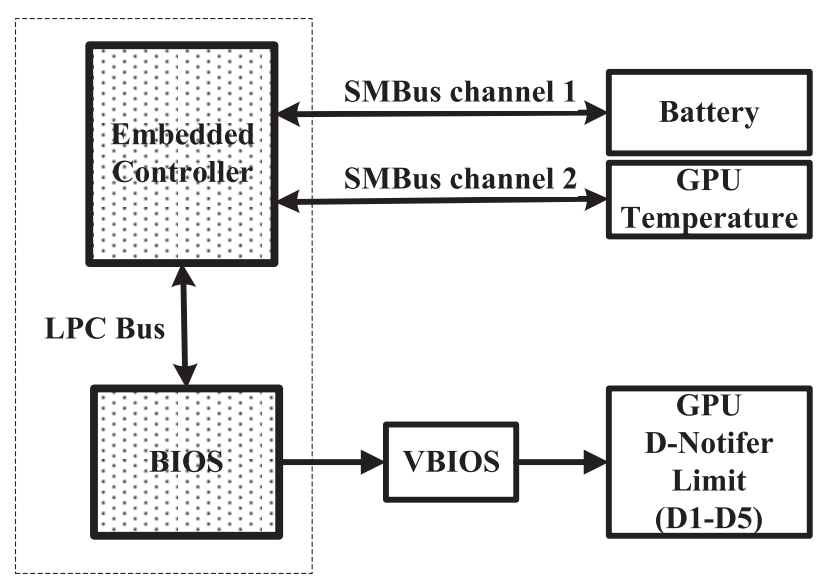

Fig. 4 The system architecture of the EC and the BIOS.

As shown in Figs. 5 (a) and 5 (b), the Voltage Regulator Module (VRM) is a device that provides a suitable supply voltage for the microprocessor. It can be soldered directly to the motherboard or can be mounted by means of a module daughter card. As it can be adjusted to adjust the supply voltage, the same motherboard can be replaced with a different supply voltage processor.

Some voltage regulators only provide a fixed supply voltage to the processor. However most voltage regulators can sense the voltage level demand from the processor.

How does the VRM supply the correct voltage to the processor? In principle, in the initial stage of the motherboard system, the VRM detects the VID (Voltage IDentificator) pin of the processor, the pin status, and the VID requirement according to the predefined check correspondence table. The voltage value first adjusts the voltage to

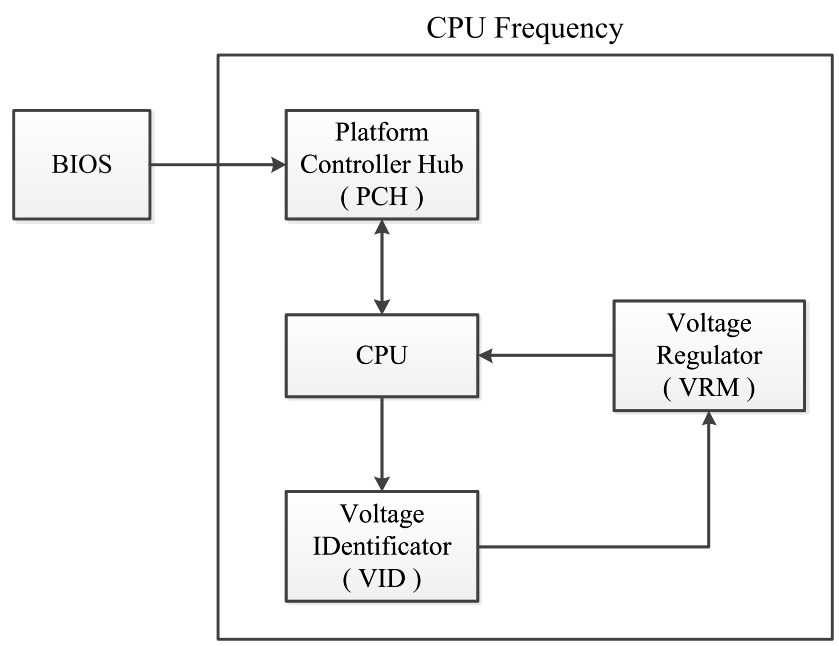

(a)

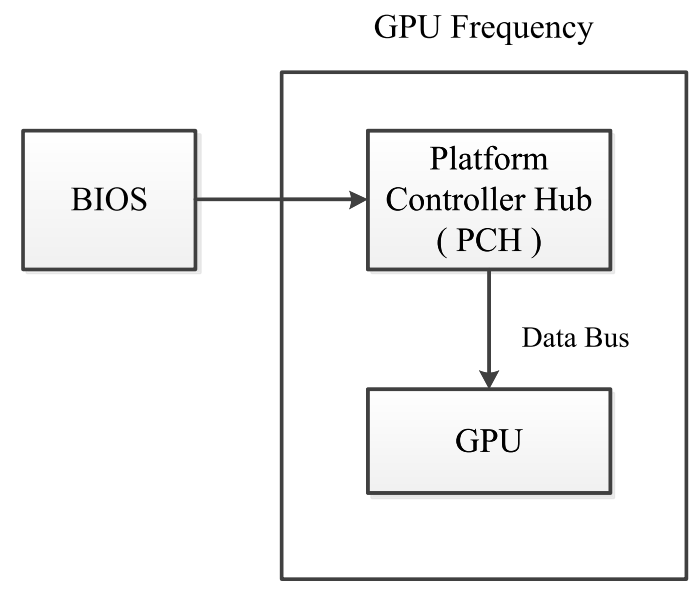

(b)

Fig. 5 (a) The hardware architecture diagram of CPU side. (b) The hardware architecture diagram of GPU side.

the voltage level required by the processor, and then supplies the voltage power to the processor. As a result, the VRM has the same role as the general voltage regulator, that is, it continues to supply power to the processor.

The VRM and the VID are currently modular hardware firmware. This paper uses the VRM and the VID modules directly, and there are no other hardware circuit designs.

Figure 6 shows the CPU and GPU frequency state transition diagram. The EC and BIOS cooperation mechanism will dynamically adjust the CPU and GPU frequency. The start conditions for the detection of the discharge " $\mathrm{C}$ " number. EC will confirm the discharge "C" number and decide whether to send a different event to the BIOS.

The term, " $\mathrm{C}$ number" is the same as the term, " $\mathrm{C}$ rating", except that this paper uses the term, " $\mathrm{C}$ number" instead of the term, " $\mathrm{C}$ rating" because some computer industries use the term, " $\mathrm{C}$ number" to indicate the term, " $\mathrm{C}$ rating".

The " $\mathrm{C}$ number" is the same as the "discharge $\mathrm{C}$ number". We will categorize the paper with the proper noun as 


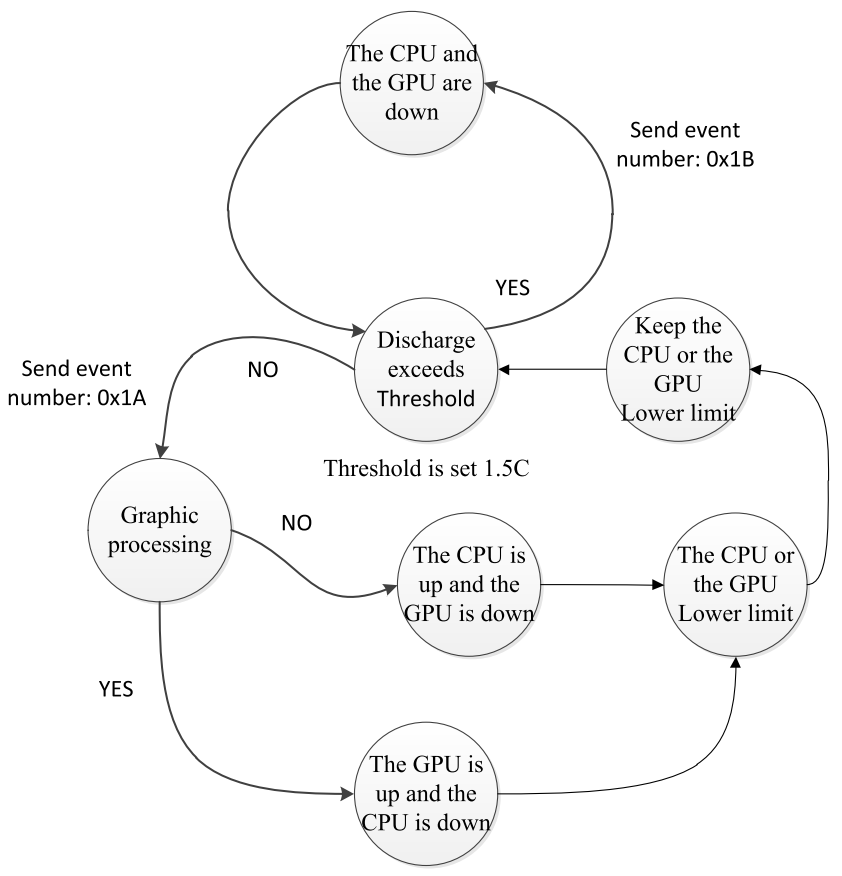

Fig. 6 Transition of CPU/GPU frequency states.

the "discharge C number".

When the "discharge $\mathrm{C}$ number" is large, as it represents the current state of the system in a high-power state of discharge, the battery body will have a hardware protection mechanism, and this protection mechanism will allow the notebook to perform the shutdown action to avoid any high power consumption. This protection mechanism affects the battery body and affects the service life of the battery itself.

The "discharge $\mathrm{C}$ number" is $1 \mathrm{C}$, which means not only that the current system power consumption is not too high, but also that it is in a normal discharge state, which has no effect on the battery or the notebook computer.

From the perspective of the notebook computer, by using the "discharge $\mathrm{C}$ number" change, we can know the power consumption status of the current notebook computer. We can control the application system according to the high or low power consumption of the system. For example: The design method of the paper dynamically adjusts the frequency of the CPU or GPU so that the value of the "discharge C number" is not large. In this way we can avoid triggering the hardware protection mechanism of the battery.

Figure 7 shows a conceptual diagram of "the CPU and the GPU are down". When the discharge is over $1.5 \mathrm{C}$, we will reduce the CPU and GPU frequency down, so that the power consumption of the whole system can be alleviated, to avoid the excessive discharge $\mathrm{C}$ number triggering the hardware protection mechanism of the battery.

Figure 8 shows the flowchart of the controlling of both the CPU and the GPU clock frequency control by using the EC and BIOS method. When the EC detects that the battery discharge "C" number is less than " $1.5 \mathrm{C}$ ", it issues an
"CPU and GPU are down" of discharge is over $1.5 \mathrm{C}$.

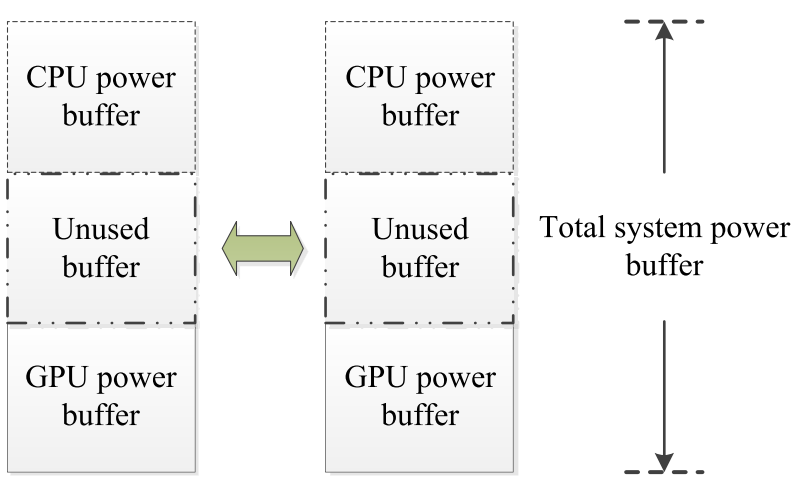

Fig. 7 "CPU and GPU are down" of discharge is over 1.5C.

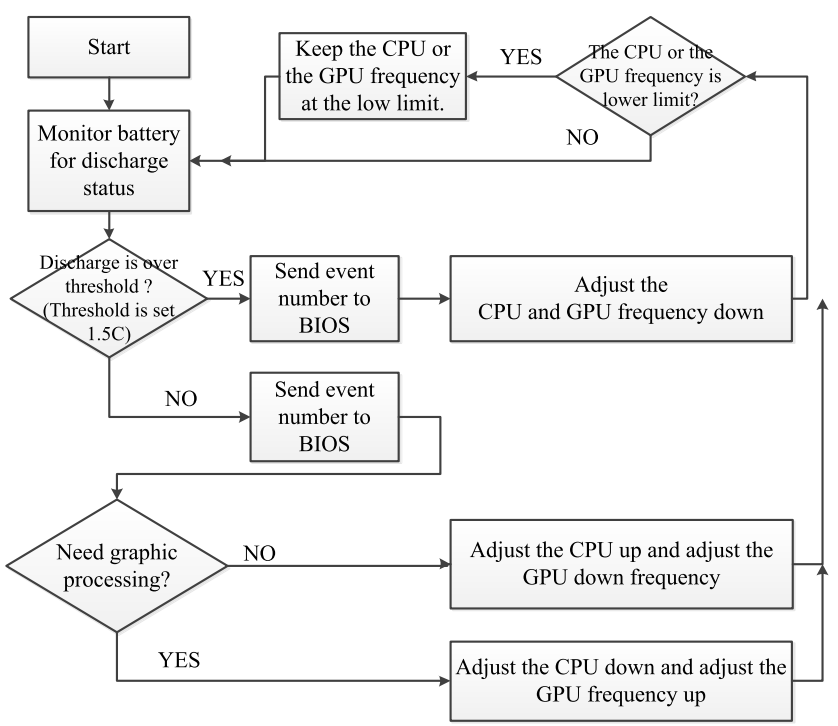

Fig. 8 Flowchart showing the CPU/GPU frequency control by using the EC and BIOS method.

"event number" to inform the BIOS to increase the clock frequency action of either the CPU or the GPU. This design can dynamically adjust the clock frequency of the CPU and the GPU in order to save power consumption to achieve the purpose of balance when the CPU is lower than the highest level of the GPU. On the contrary, when the CPU is higher than the GPU, then the CPU is lowered. When the EC detects that the battery discharge "C" number is greater than "1.5C", it then issues another "event number" to notify the BIOS to carry out either the proper CPU or GPU down-frequency action.

The EC will detect the battery status by polling $100 \mathrm{~ms}$. If you need to adjust the CPU/GPU frequency, it will immediately issue an interrupt command to the BIOS.

The frequency change between the CPU and the GPU is adjusted in a level manner. For example: if the CPU frequency range is $0.8 \mathrm{GHz}-3.2 \mathrm{GHz}$, we divide this range into 12 levels. Each level is increased or decreased by $0.2 \mathrm{GHz}$. 
The following pseudo-code represents the sensing of the discharge "C" number and the control of the CPU/GPU cooperation by redesigning the EC/BIOS firmware program.

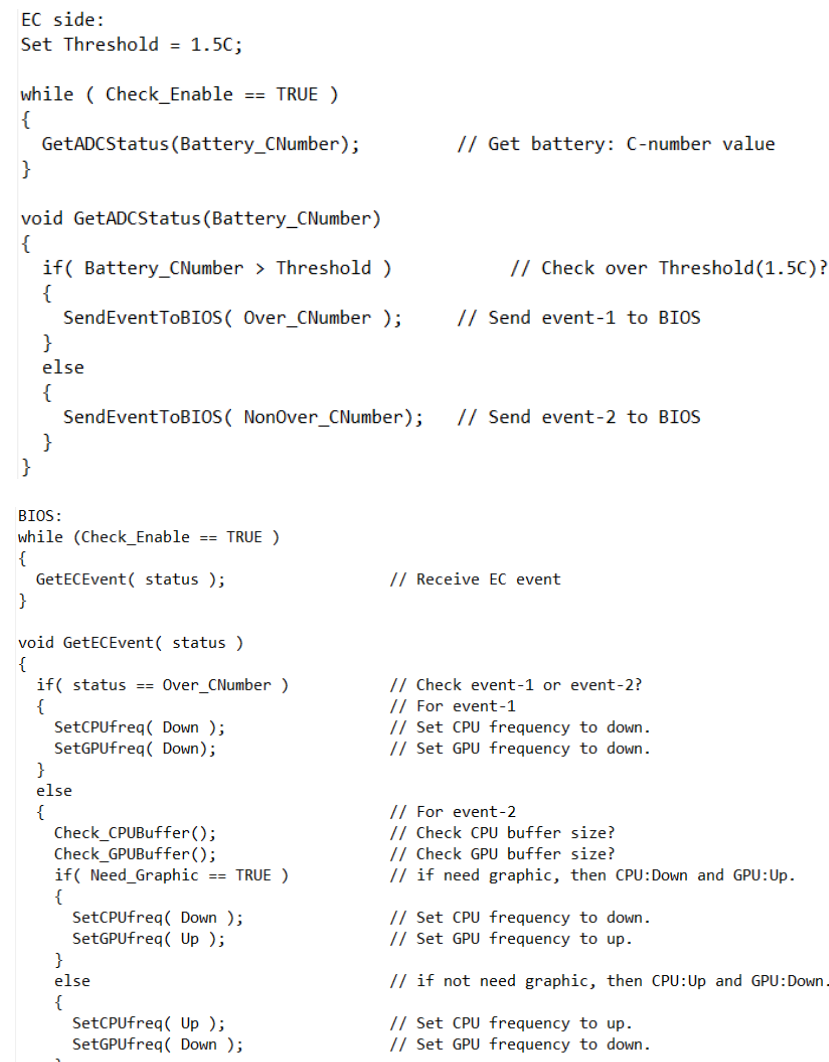

The main modules consist of four parts: EC, BIOS, CPU, and GPU modules. To enhance the efficiency, the EC firmware program uses assembly language. The BIOS firmware program uses $\mathrm{C}$ language. The speed of the interrupt mode is calculated in "ns" units. The notification from the EC to the BIOS to complete the entire frequency range of the whole operation time takes only about less than $1 \mathrm{~ms}$ to complete, because the EC uses its interrupt function to inform the BIOS to complete the ups and downs of the clock frequency. This notification will not result in any frame lag phenomenon during a high resolution video game.

The power consumption of the low power EC is about $2-3 \mathrm{~mW}$, which is very small in comparison with the total power consumption of the whole system.

The cooperation mechanism between the BIOS and the EC can balance the system power consumption and the system performance between the CPU and the GPU. The EC is responsible for detecting the system power consumption and the battery discharge " $\mathrm{C}$ " number. The BIOS is responsible for balancing the performance both of the CPU and of the GPU.

The response time of the EC detection is about $5 \mathrm{~ms}$. The issue time from an interrupt signal of the EC to the BIOS is about $1 \mathrm{~ns}$. And then as the BIOS takes 1-2 ms to dynamically adjust the performance of the CPU and the
Dynamic adjustment CPU and GPU power consumption

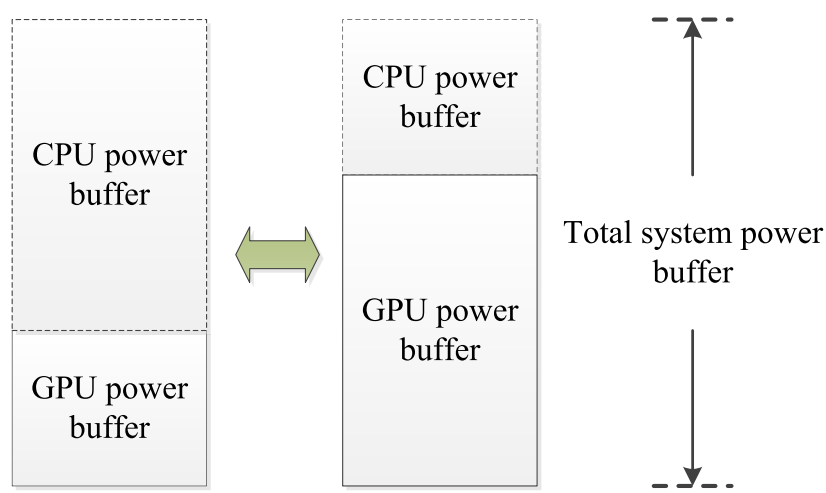

Fig.9 Dynamic adjustment CPU and GPU power consumption.

GPU, the overall dynamic adjustment operation takes about 6-7 ms to complete a cycle.

This design uses the EC to detect the operation condition of the gaming notebook and then to notify the BIOS to perform interrupt instructions to balance and to adjust the performance of the CPU and the GPU. To maintain an sufficient performance of the GPU for high resolution image processing performance, this design provides an easier way to achieve the balance between the system power consumption and the system performance of both the CPU and the GPU [19]-[22].

These control actions will be based on the battery discharge "C" number issuance at any time; the main purpose is to set the system discharge limit at " $1.5 \mathrm{C}$ ". The definition of the discharge standard is "1.5C" which depends on the battery itself. The maximum discharge " $\mathrm{C}$ " number is used to make any adjustment. Of course, different batteries may set a different discharge " $\mathrm{C}$ " number. Each discharge " $\mathrm{C}$ " number and the capacity of the battery have a close relationship. This relationship can be used to estimate the charging time, the discharge time and the discharge capacity. For example: a 3S 2200mAh lithium polymer battery, its 1 " $\mathrm{C}$ " is $2.2 \mathrm{~A}$. We first apply the formula $2200 \mathrm{mAh} / 1000=2.2 \mathrm{Ah}$, and then remove the "h" to obtain $2.2 \mathrm{~A}$. 2 "C" is $2 \times 2.2 \mathrm{~A}=4.4 \mathrm{~A}$.

Figure 9 shows the dynamic adjustment of the CPU and the GPU power consumption. If the system's power buffer is fixed, this design uses complementary adjustments both of the CPU and of the GPU power consumption. When the CPU power consumption decreases, as the power buffer will be moved to the GPU, then the GPU can increase the power consumption. Conversely, when the GPU power consumption decreases, the CPU can increase the power consumption. By utilizing this way, the system can control the total power consumption of the system, and then this design will automatically adjust the performance to reach a preset level.

Due to the special physiological factor of the human eye, if the video frame is updated at a rate higher than about 10-12 FPS, a video frame can be interpreted by human eyes 
to be a continuous video. This phenomenon is called visual persistence. But, for shooting video games or racing video games, if the frame update rate is less than 30 FPS, then this video game will appear to be discontinuous, which is commonly known as frame lag phenomenon. This phenomenon is called visual persistence.

When the operation conditions are met, the CPU and the GPU performance drop will have a certain limit, because this design must maintain a certain level of performance, so the minimum limit of the CPU and the GPU performance will not be further reduced.

This design can maintain 30 FPS for the $4 \mathrm{~K}$ video game as a benchmark. The CPU and the GPU need to adjust the discharge "C" number of settings in advance in order to maintain 30 FPS which is required for conducting experiments. As this design presets the value of the discharge " $\mathrm{C}$ " number, the range discharge " $C$ " number can be determined after the start of the dynamically adjust mechanism, which can be used in a follow-up to make some minor adjustments.

Because the battery mode of the previous designs limits the battery discharge " $\mathrm{C}$ " number, the battery mode has only half of the performance which may result in the frame lag phenomenon. As this design eliminates the limitation of the battery discharge " $\mathrm{C}$ " number in the battery mode, this design can enhance the performance like that of the AC mode. Therefore, this design must detect the battery discharge "C" number. When the battery discharge "C" number is too large, this design will provide the battery protection. Overall, the EC detection and the BIOS control can dynamically adjust the system performance and the power consumption, and then provide the enough performance for the high resolution image processing and achieve the required balance.

This design uses the battery discharge "C" number as a monitoring target because the discharge " $\mathrm{C}$ " number is commonly used for the gaming notebook battery. The lowpower embedded controller (EC) monitors the discharge "C" number in the gaming notebook battery which allows the system to maximize the performance of the gaming notebook battery mode.

The dynamic adjustment of the CPU/GPU implementation is found in many papers, but this paper uses a lowpower EC to dynamically adjust the CPU/GPU which is an easier software control design than other designs, so that the gaming notebook can maintain a high performance in battery mode.

The idea of this design is simple and fast. This design does not require any complicated circuit and algorithm. This design uses the existing hardware architecture directly and serves as the new hardware architecture controlled by the redesign EC/BIOS firmware program.

Without using excessively complicated hardware circuits, the monitoring of the discharge " $\mathrm{C}$ " number can be used to sense the status of the gaming notebook in the battery mode, and then the redesign firmware can be used to control the CPU/GPU cooperation which is both easier and faster than other designs to achieve the necessary perfor- mance adjustment in the battery mode.

\section{Experiment Results}

Tables 2-5 shows the specifications for our gaming notebook, common notebook, other low end gaming notebook, and other high end gaming notebook.

As shown in Tables 2-5, we have tested four kinds of the gaming notebooks with three games: GTA5, TR2013, and BioShock which are as shown in Fig. 10. During the game test, we have recorded the experimental data and

Table 2 Specifications for a gaming notebook experiment.

\begin{tabular}{cc}
\hline Platform & Modern Micro-architecture Bridge \\
\hline CPU & Quad-Core Mobile CPU (3.7GHz, TDP:95W) \\
GPU & GDDR5(8G) MXM III (TDP:60W 150W) \\
RAM & DDR4-2133 8GB*4 \\
HDD & SATA III SSD 512GB \\
Panel & 15.6-inch QFHD(3840x2160) (LED PANEL) \\
\hline
\end{tabular}

Table 3 Specifications for a common notebook experiment.

\begin{tabular}{cc}
\hline Platform & Modern Micro-architecture Bridge \\
\hline CPU & Quad-Core Mobile CPU (1.8GHz, TDP:15W) \\
GPU & GDDR5(8G) MXM III (TDP:60W 150W) \\
RAM & DDR4-2133 8GB2 \\
HDD & SATA III SSD 512GB \\
Panel & 15.6-inch QFHD(3840x2160) (LED PANEL) \\
\hline
\end{tabular}

Table 4 Specifications for an other low end gaming notebook experiment.

\begin{tabular}{cc}
\hline Platform & Modern Micro-architecture Bridge \\
\hline CPU & Quad-Core Mobile CPU (3.1GHz, TDP:65W) \\
GPU & GDDR5(8G) MXM III (TDP:60W 150W) \\
RAM & DDR4-2133 8GB*4 \\
HDD & SATA III SSD 512GB \\
Panel & 15.6-inch QFHD(3840x2160) (LED PANEL) \\
\hline
\end{tabular}

Table 5 Specifications for an other high end gaming notebook experiment.

\begin{tabular}{cc}
\hline Platform & Modern Micro-architecture Bridge \\
\hline CPU & Quad-Core Mobile CPU (3.7GHz, TDP:95W) \\
GPU & GDDR5(8G) MXM III (TDP:60W 150W) \\
RAM & DDR4-2133 8GB*4 \\
HDD & SATA III SSD 512GB \\
Panel & 15.6-inch QFHD(3840x2160) (LED PANEL) \\
\hline
\end{tabular}

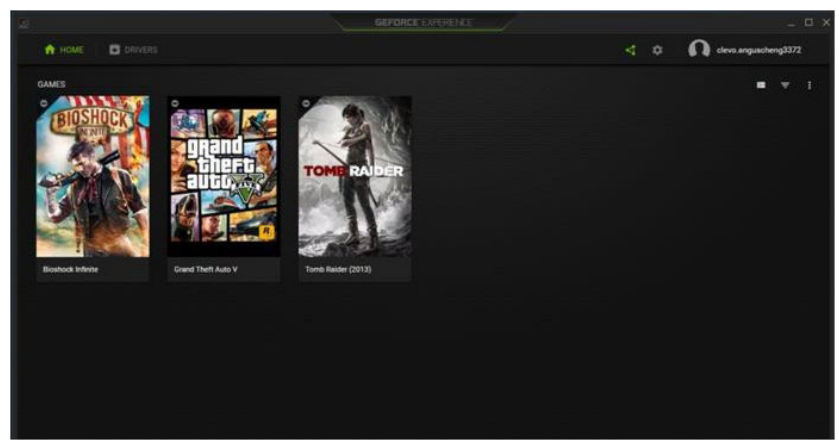

Fig. 10 The three test games. 


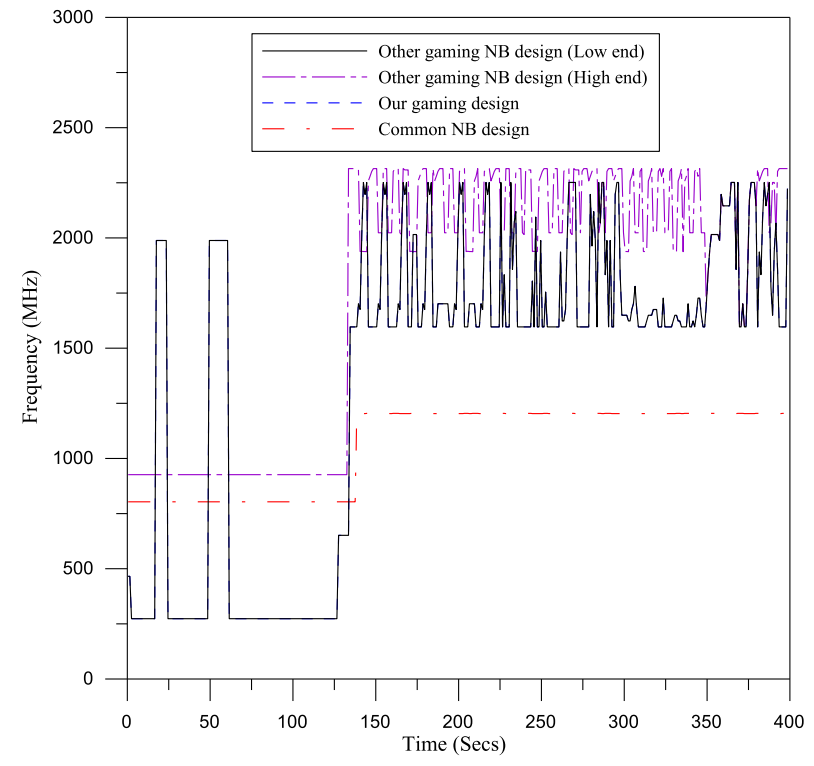

Fig. 11 The CPU frequency of different designs.

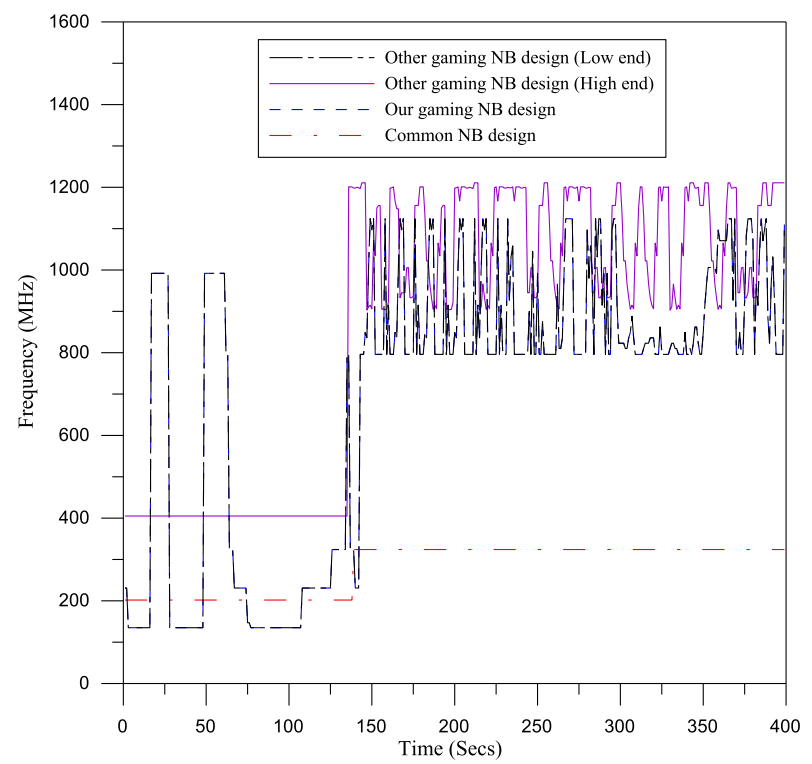

Fig. 12 The GPU frequency of different designs.

shown them in Figs. 11-22. Some of the experimental data are averaged.

Figure 11 and Fig. 12 show the measurement of the CPU and the GPU clock frequency change. Figure 10 shows the measurement of four different notebook CPU clock frequency changes. To save power consumption in the battery mode, the common notebook design will decrease the CPU clock frequency directly, so the CPU will remain at a low speed. Other notebook designs, although they do not directly decrease the CPU clock frequency to a low speed, will still limit the CPU clock frequency.

Based on the maximum discharge "C" number of the specific system, this design will increase the CPU clock frequency to meet the limit of the power consumption from the maximum discharge "C" number, so that the average $\mathrm{CPU}$ clock frequency of this design is higher than the other designs in order to maintain a sufficient performance without any frame lag phenomenon.

Figure 12 shows a measurement of four different notebook GPU clock frequency variations which are the result of the operation shown in Fig. 10, the CPU operation measurement; this design model presents a high performance when used in a high speed and a high resolution video game.

From the measurement, when this design controls the CPU clock frequency at $1600 \mathrm{MHz}-2200 \mathrm{MHz}$, the other designs, which may stay at $1300 \mathrm{MHz}-1500 \mathrm{MHz}$ or $800 \mathrm{MHz}-$ $1200 \mathrm{MHz}$, is not a sufficient performance. In addition, when this design controls the GPU clock frequency at the $800 \mathrm{MHz}-1100 \mathrm{MHz}$, the other designs, which may stay at the $400 \mathrm{MHz}-750 \mathrm{MHz}$ and $200 \mathrm{MHz}-300 \mathrm{MHz}$, may have a frame lag phenomenon when running a high resolution video game.

This system is based on the CPU and GPU power buffer to dynamically adjust the clock frequency. If the CPU power consumption reduces, the total power buffer will increase and then transfer more power to the GPU, by which the GPU clock frequency will be increased. On the contrary, if the GPU power consumption reduces, the total power buffer will increase and then transfer more power to the CPU, by which the CPU clock frequency will be increased. In this design, after several measurements and fine tuning have been carried out, the total buffer is determined by whether or not it exceeds " $1.5 \mathrm{C}$ ".

The four designs, i.e., the common notebook, this gaming notebook, the low end gaming notebook, and the high end gaming notebook are tested by utilizing the 3D game separately. All four designs are all recorded in the FPS and are shown in Fig. 13, Fig. 14, Fig. 15 and Fig. 16 respectively. In the process of running the game, a FPS of less than 30 is likely to cause a frame lag phenomenon. From the data in Figs. 13, 14, 15 and 16, as the performance of the common notebook is almost all lower than 20 FPS, the frame lag phenomenon is very serious. The frame lag phenomenon percentage of the total running time is about $50 \%-60 \%$. In the other gaming notebook in the course of the game there are some parts of less than 30 FPS, with a frame lag phenomenon percentage of about $10 \%-15 \%$. This design of the gaming notebook is shown in Fig. 13, which maintains more than 30 FPS, with a frame lag phenomenon percentage of $0 \%$.

From our experimental measurements, this design, with an automatically dynamic adjustment of the CPU and the GPU clock frequency, can maintain a sufficient performance in the course of a high resolution video game at 30 FPS. Our experimental measurement shows that there is no game frame lag phenomenon. In contrast to the common notebook, many time points have occurred in the case of the frame lag phenomenon, which is less than 30 FPS.

Figure 17 and Fig. 18 shows the measurement of the power consumption of the CPU and the GPU. The measurements show the CPU clock frequencies are changing but not 


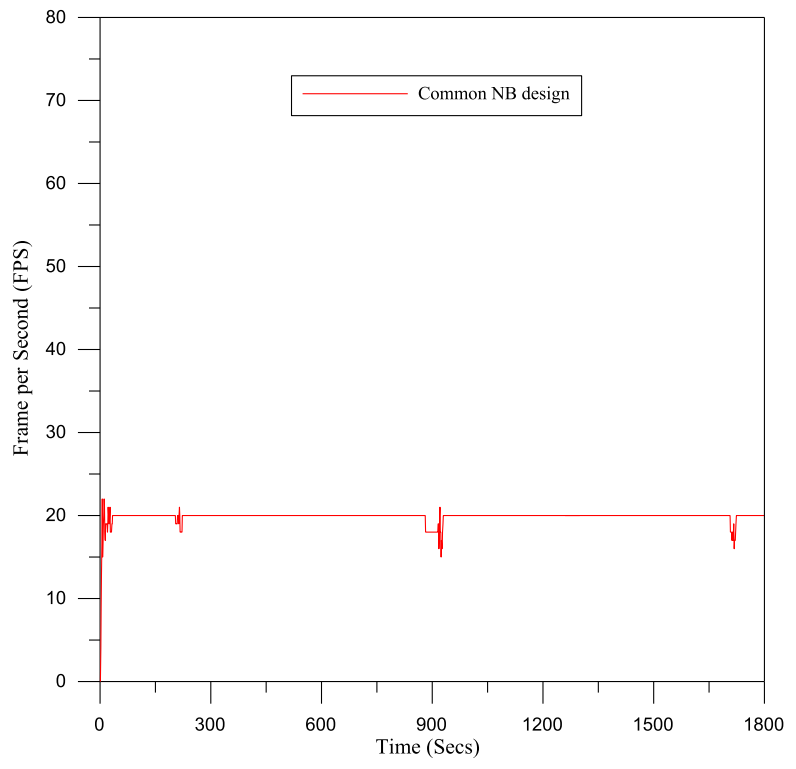

Fig. 13 The FPS of a common notebook design.

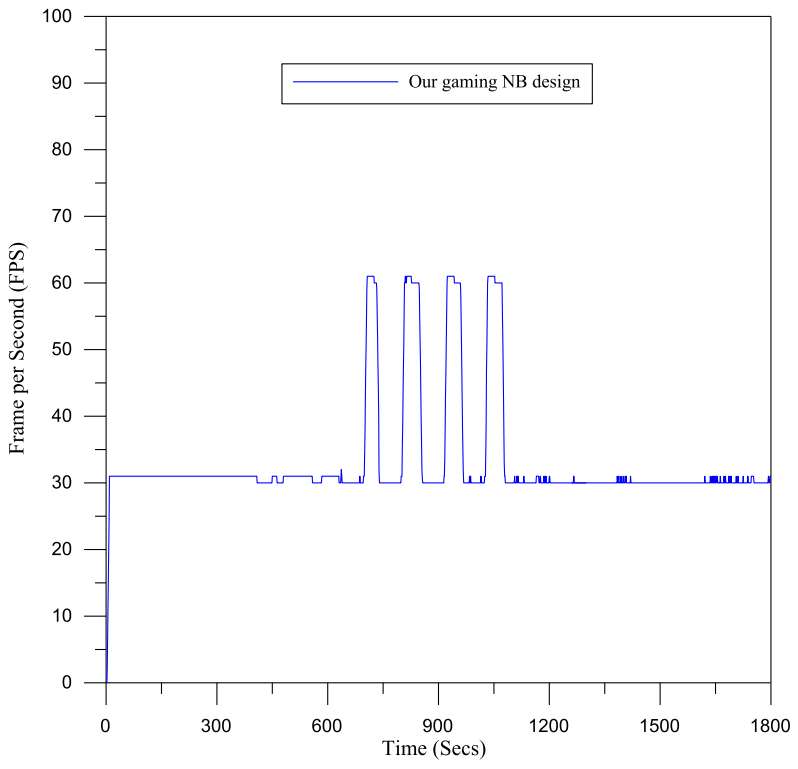

Fig. 14 The FPS with a 1.5C of our gaming notebook design.

very much in comparison with the GPU. The main purpose of this design is to allow the GPU to have a greater changing space to meet the needs of a high resolution video game, which is shown as Fig. 17, the power consumption of the GPU.

Figure 19 shows the range of power consumption for the CPU and the GPU in this system. When the CPU power consumption is at $24 \mathrm{~W}-26 \mathrm{~W}$, the corresponding GPU power consumption will be about $110 \mathrm{~W}-97 \mathrm{~W}$ with the normal complementary variation. Conversely, when the CPU power consumption rose to $27 \mathrm{~W}-30 \mathrm{~W}$, the GPU power consumption will be reduced to $92 \mathrm{~W}-85 \mathrm{~W}$. This design provides the balance adjustment action of the power consumption ac-

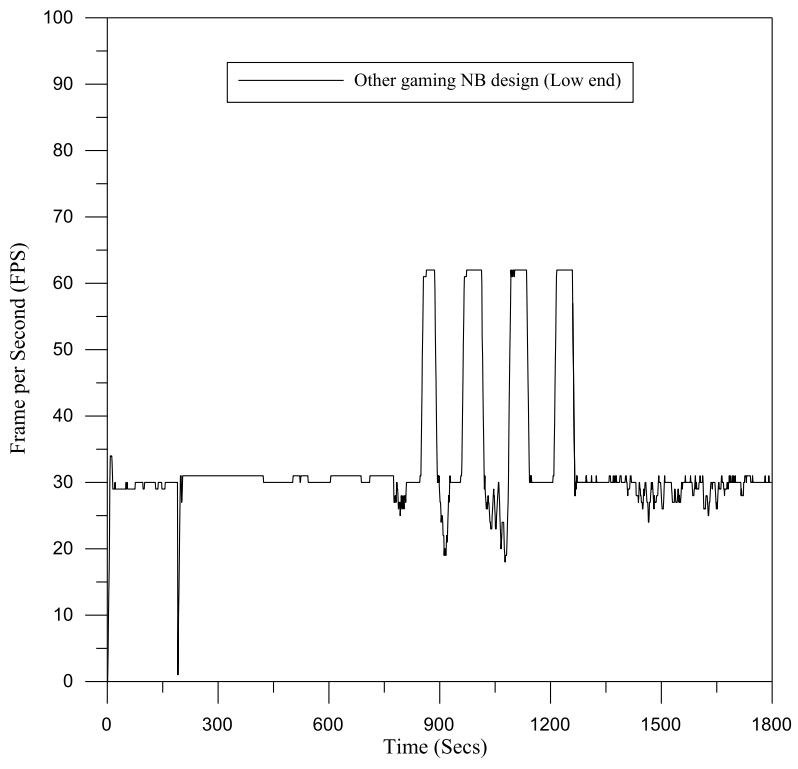

Fig. 15 The FPS of a low end gaming notebook design.

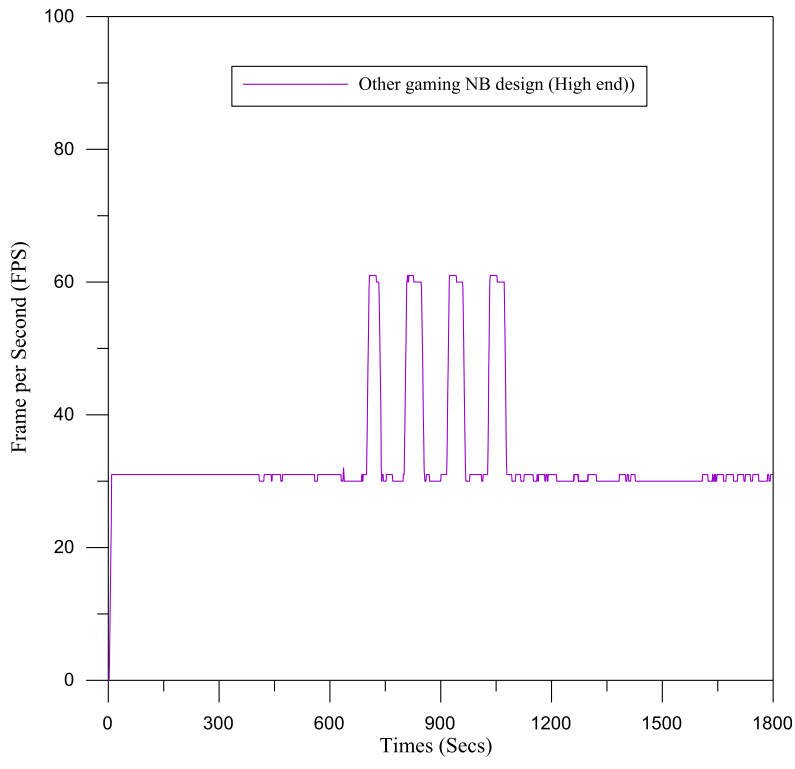

Fig. 16 The FPS of a high end gaming notebook design.

cording to the maximum discharge "C" number of the battery.

The discharge " $C$ ' number in Fig. 19 is the variable discharge " $\mathrm{C}$ ' number which is not the fixed threshold setting of the discharge " $C$ " number.

Figure 20 shows four measurements of the total power consumption of the CPU and the GPU among the four gaming notebook designs.

Figure 21 shows another experiment which was run 10 times to record the measurement curves of the battery life of four designs. The curve of this design in Fig. 19 shows that the improved performance may sometimes sacrifice the battery life. But, in comparison with high end gaming note- 


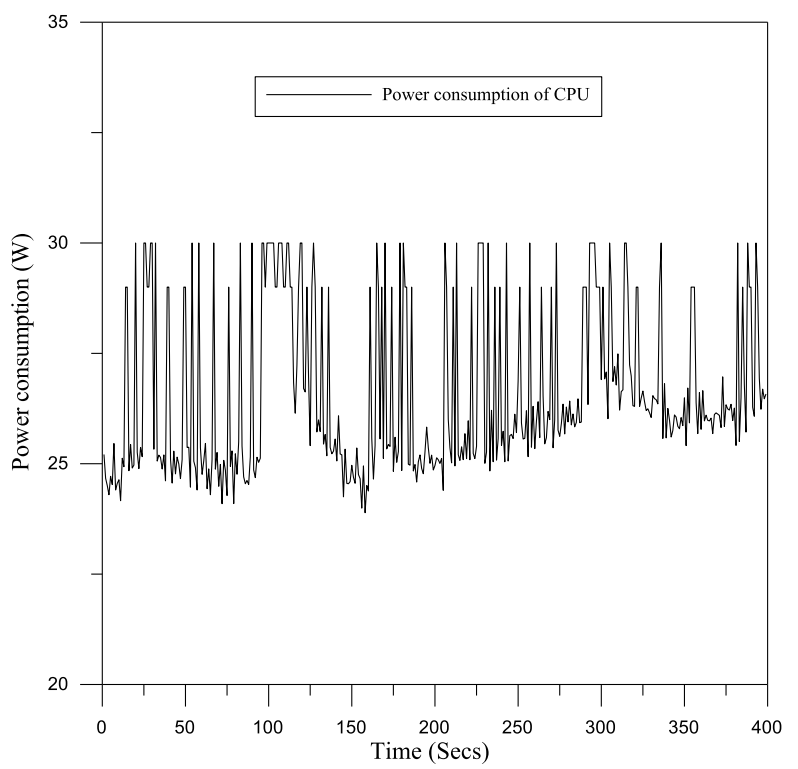

Fig. 17 The power consumption of the CPU.

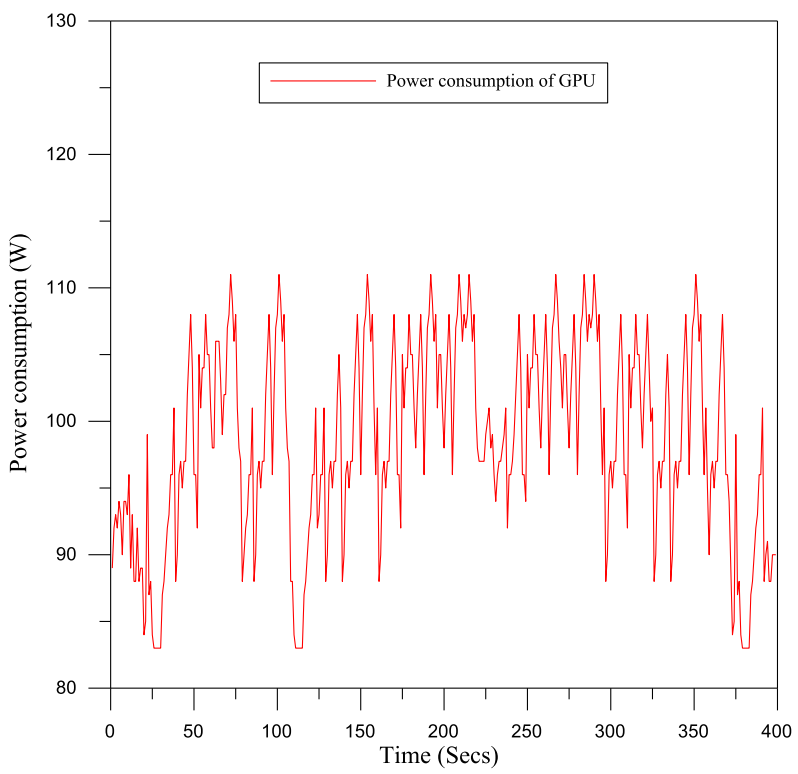

Fig. 18 The power consumption of the GPU.

books, this design provides a sufficient performance for a high resolution video game with a lesser power consumption due to the automatically dynamic adjustment of both the CPU and the GPU clock frequency.

Figure 22 shows the 3DMark score of different designs. The common design was dropped from 13500 to 14000 because it was directly down-converted in a battery mode. This design with the battery mode runs at the score of from 17500 to 18000 which provide a sufficient performance for a high resolution video game. Therefore, within the limits of the number of battery discharges, this design dynamically adjusts both the CPU and the GPU clock frequency so that the high speed and high resolution display can maintain almost

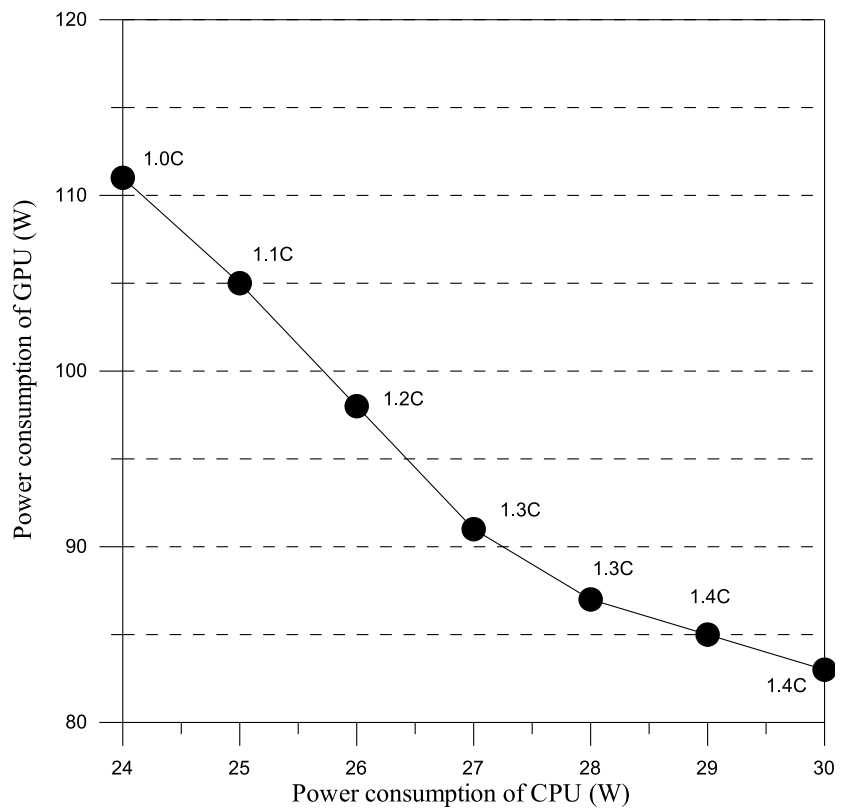

Fig. 19 The range of power consumption for the CPU and the GPU.

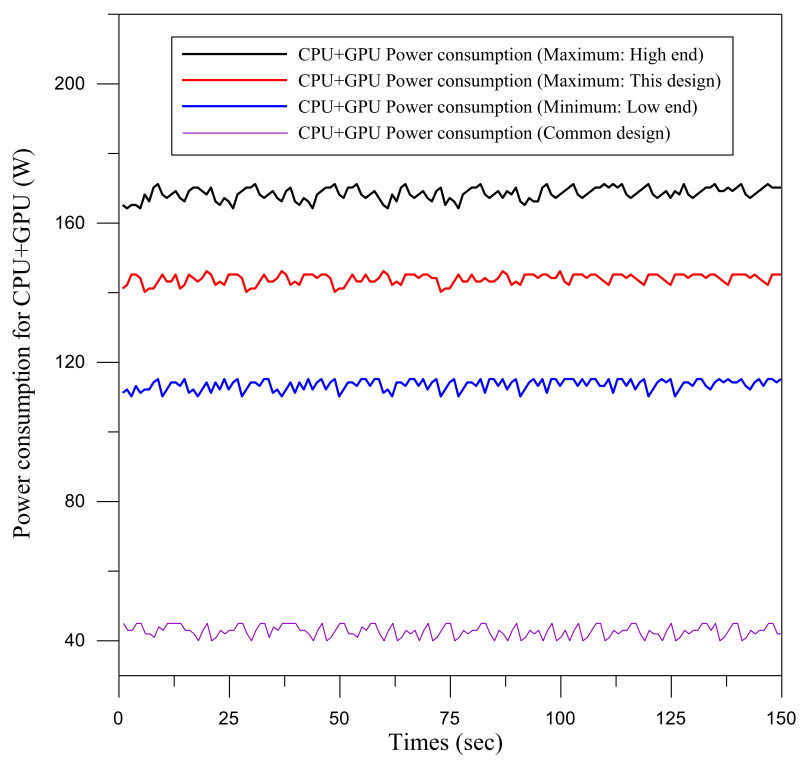

Fig. 20 The power consumption for the CPU and the GPU of the four gaming notebook designs.

same performance as the $\mathrm{AC}$ mode of the gaming notebook.

Table 6 shows the comparison table of the four designs. This design emphasizes a balance of both the performance and the power consumption. To maintain a sufficient level, this design may consume certain power and thus needs to sacrifice some battery life. However this design still saves some power consumption in comparison with a high end gaming notebook. Based on the maximum battery discharge "C" number, this design automatically dynamically adjusts both the CPU and the GPU clock frequency and maintains a sufficient level of the performance which is better than that of a low end notebook. 


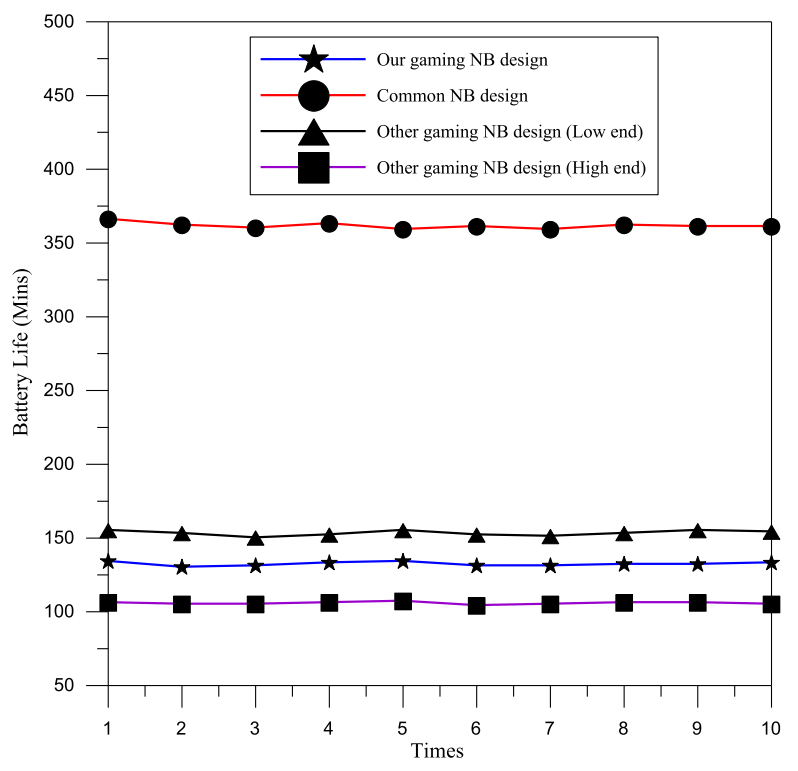

Fig. 21 The battery life of different notebook designs.

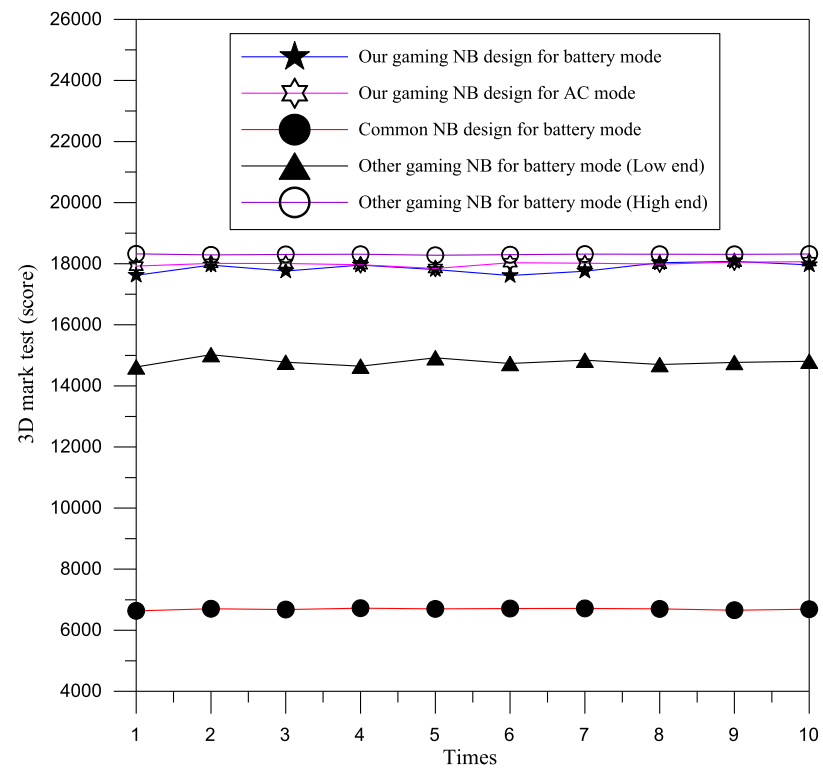

Fig. 22 Run 3DMark score of different notebook designs.

When this design is tested by utilizing 3D games, it obtains a frame lag percentage of $0 \%$ in comparison with a 50-60\% frame lag percentage of a common notebook and a $10-15 \%$ frame lag percentage of a low end notebook. Although a high end gaming notebook can also have a frame lag percentage of $0 \%$, as it consumes more power, it has a short battery life in comparison with our design.

This design cost is high, but it is lower than that of the high end gaming notebook. The main cost difference is the need for an AC adapter and a battery.

For the "Frame Lag Percentage" and "Average Battery Life" two data, with the relative comparison of an indicator can be more clearly seen in the difference between the four different designs. Equation (1) and Eq. (2) show the rela-
Table 6 A comparison of the other designs and our design.

\begin{tabular}{ccccc}
\hline & $\begin{array}{c}\text { Common } \\
\text { NB } \\
\text { design }\end{array}$ & $\begin{array}{c}\text { Other } \\
\text { gaming NB } \\
\text { design } \\
\text { (Low end) }\end{array}$ & $\begin{array}{c}\text { Other } \\
\text { gaming NB } \\
\text { design } \\
\text { (High end) }\end{array}$ & $\begin{array}{c}\text { Our } \\
\text { gaming } \\
\text { NB } \\
\text { design }\end{array}$ \\
\hline $\begin{array}{c}\text { Dynamic control of } \\
\text { the CPU and GPU } \\
\text { frequency. }\end{array}$ & No & Yes & Yes & Yes \\
\hline Keep 30 FPS. & No & No & Yes & Yes \\
\hline $\begin{array}{c}\text { Battery mode } \\
\text { Performance of } \\
\text { system. }\end{array}$ & Low & Medium & High & High \\
\hline $\begin{array}{c}\text { Performance gap } \\
\text { between the AC mode } \\
\text { and the Battery mode } \\
\text { is little. }\end{array}$ & No & No & Yes & Yes \\
\hline Frame lag percentage & $50 \%-60 \%$ & $10 \%-15 \%$ & $0 \%$ & $0 \%$ \\
\hline $\begin{array}{c}\text { Average for Battery } \\
\text { life. }\end{array}$ & 361 mins & 153 mins & 105 mins & 132 mins \\
\hline Design cost & Low & High & High & High \\
\hline
\end{tabular}

tive comparison index of the "Frame Lag Percentage" of the different designs:

A: Common notebook design

B: Other low end gaming notebook design

C: Other high end gaming notebook design

D: Our gaming notebook design

$$
\begin{aligned}
& (\mathrm{B} / \mathrm{A}) \times 100 \% \\
& (\mathrm{D} / \mathrm{A}) \times 100 \%
\end{aligned}
$$

$\mathrm{A}$ and $\mathrm{B}$ has the following relative comparison index of the Frame Lag Percentage: $(15 / 60) \times 100 \%=25 \%$

$\mathrm{A}$ and $\mathrm{D}$ has the following relative comparison index of the Frame Lag Percentage: $(0 / 60) \times 100 \%=0 \%$

Since this new design has the smaller relative comparison index value of the Frame Lag Percentage, this new design is better for a gaming notebook design.

Equation (3) and Eq. (4) show the "Average Battery Life" of different designs:

$$
\begin{aligned}
& (\mathrm{C} / \mathrm{A}) \times 100 \% \\
& (\mathrm{D} / \mathrm{A}) \times 100 \%
\end{aligned}
$$

$\mathrm{A}$ and $\mathrm{C}$ have the relative comparison of the indicator of the Average Battery Life: $(105 / 361) \times 100 \%=29 \%$

$\mathrm{A}$ and $\mathrm{D}$ have the relative comparison of the indicator of the Average Battery Life: $(132 / 361) \times 100 \%=36.5 \%$

Since this new design has the larger relative comparison of the indicator value of the Average Battery Life, this new design has a longer battery recharge cycle in the gaming notebook design.

To compare the four gaming notebooks, we use three games: GTA5, TR2013, and BioShock. We use the 200 seconds image length to record "the Standard Deviation of the Frame Lag Percentage" and "the Mean of the Frame Lag Percentage". Every 10 seconds a statistical time interval unit, measures "the Mean of the Frame Lag Percentage" and "the Standard Deviation of the Frame Lag Percentage" of the game images of 200 seconds. For 20 statistical time interval units, there are 20 samples. Each sample contains 10 seconds of the game image Frame Lag. Table 7 shows the 200-second images calculation of the game for the "the 
Table 7 A comparison table for the Frame Lag Percentage of the four designs of the gaming notebooks.

\begin{tabular}{|c|c|c|c|}
\hline & GTA5 & TR2013 & BioShock \\
\hline $\begin{array}{l}\text { The Mean of the Frame Lag Percentage } \\
\text { (Common notebook) }\end{array}$ & 9.5 & 9.48 & 9.45 \\
\hline $\begin{array}{l}\text { The Standard Deviation of the Frame Lag } \\
\text { Percentage (Common notebook) }\end{array}$ & 0.45 & 0.4484 & 0.4475 \\
\hline $\begin{array}{l}\text { The Mean of the Frame Lag Percentage } \\
\text { (Other low-end gaming notebook) }\end{array}$ & 5.2 & 4.8 & 4.7 \\
\hline $\begin{array}{c}\text { The Standard Deviation of the Frame Lag } \\
\text { Percentage } \\
\text { (Other low-end gaming notebook) }\end{array}$ & 0.511 & 0.36 & 0.3515 \\
\hline $\begin{array}{l}\text { The Mean of the Frame Lag Percentage } \\
\text { (Other high-end gaming notebook) }\end{array}$ & 0.1 & 0.15 & 0.15 \\
\hline $\begin{array}{c}\text { The Standard Deviation of the Frame Lag } \\
\text { Percentage } \\
\text { (Other high-end gaming notebook) }\end{array}$ & 0.1295 & 0.1275 & 0.1275 \\
\hline $\begin{array}{l}\text { The Mean of the Frame Lag Percentage } \\
\text { (Our gaming notebook) }\end{array}$ & 0.15 & 0.15 & 0.15 \\
\hline $\begin{array}{c}\text { The Standard Deviation of the Frame Lag } \\
\text { Percentage } \\
\text { (Our gaming notebook) }\end{array}$ & 0.1625 & 0.1625 & 0.1625 \\
\hline
\end{tabular}

Standard Deviation of the Frame Lag Percentage" and "the Mean of the Frame Lag Percentage".

Equation (5) shows "the Mean of the Frame Lag Percentage".

$$
\bar{x}=\frac{1}{N} \sum_{i=1}^{N} x_{i},(N=20)
$$

Equation (6) shows "the Standard Deviation of the Frame Lag Percentage".

$$
\sigma=\sqrt{\frac{1}{N} \sum_{i=1}^{N}\left(x_{i}-\bar{x}\right)^{2}},(N=20)
$$

Table 7 shows "the Mean of the Frame Lag Percentage" and "the Standard Deviation of the Frame Lag Percentage" of the three test games with the 200-second images. Our gaming notebook has the similar high resolution video performance as the high-end gaming notebook, though our design consumes less power and runs the high resolution video game for a longer time as shown in Table 6 .

When the discharge $\mathrm{C}$ number exceeds $1.5 \mathrm{C}$, the total power consumption of the current system has exceeded the upper limit of the battery body. At this time, if the discharge $\mathrm{C}$ number continues to rise, the battery will then start the hardware protection mechanism. This protection mechanism will cause the hardware circuit inside the battery to be powered off instantaneously to prevent the line from being abnormally burned. When the battery is powered off, the battery cannot be used to provide power to the notebook, and the entire system will be shut down.

If the discharge $\mathrm{C}$ number does not exceed $1.5 \mathrm{C}$, it means that the total power consumption of the current system is in the state that can maintain the battery in normal use, which is a state that is normal for the system or the battery.

Therefore, if the discharge $\mathrm{C}$ number exceeds $1.5 \mathrm{C}$, this design must reduce the power buffer of the CPU and the GPU, so that the system has sufficient buffer time, and as

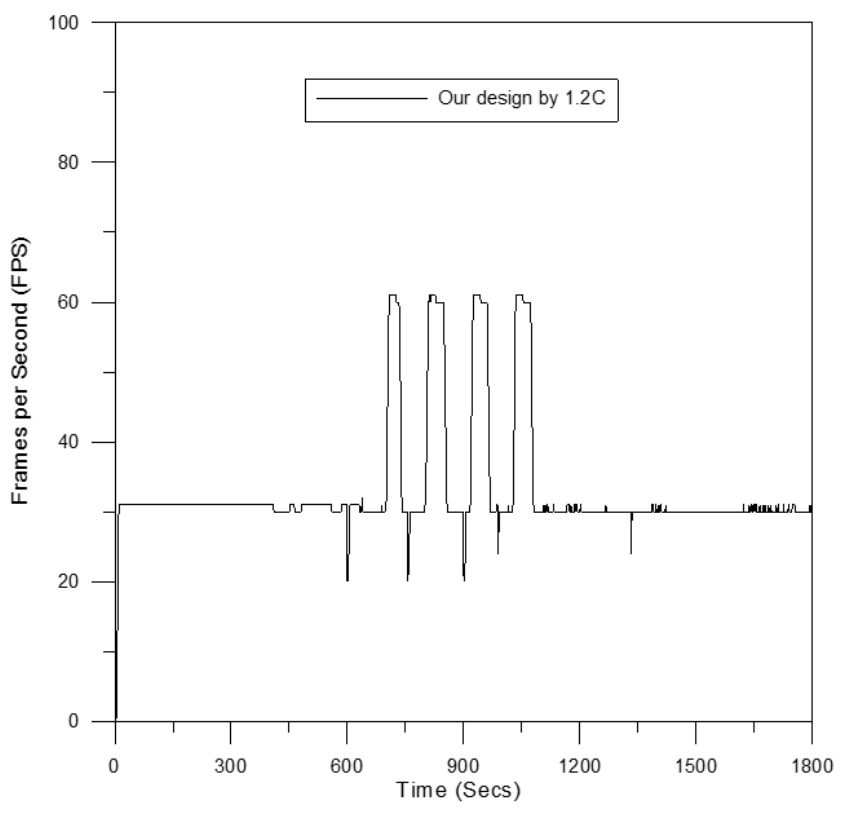

Fig. 23 The FPS with a $1.2 \mathrm{C}$ of our gaming notebook design.

this falling action will cause the FPS to be lower than 30 , and the Frame Lag phenomenon will then occur.

In the battery mode, the power consumption of the gamming notebook can be monitored and judged by the discharge " $\mathrm{C}$ " number which represents the ratio of the power consumption.

In the second chapter of this paper, we explain the meaning of the discharge " $\mathrm{C}$ " number and how to monitor the amount of the current that the battery is discharging. This discharge "C" number can also be used as an indicator of the gamming notebook power consumption in the battery mode.

To dynamically adjust and control the CPU/GPU frequency, this design uses an existing gamming notebook motherboard via the new EC/BIOS cooperation program. There is no need for extra complicated hardware chips or algorithms. What we are pursuing is simply to quickly achieve the goal by using the new EC/BIOS cooperation program.

We tested a total of four parameters from $1.2 \mathrm{C}$ to $1.5 \mathrm{C}$ as shown in Fig. 23, Fig. 24, Fig. 25 and Fig. 14 respectively. We found that $1.5 \mathrm{C}$ works best with respect to the frame lag of the video performance of the gamming notebook.

The $1.5 \mathrm{C}$ threshold is not calculated by a set of formulas. The $1.5 \mathrm{C}$ threshold designed in this paper is the resulting data of $1.5 \mathrm{C}$ which is much better after conducting many experiments. Depending on the battery capacity or the cell, this $1.5 \mathrm{C}$ may need to be fine-tuned, but we don't think this change will take place often. We will implement the fine turning from between $1.2 \mathrm{C}$ and $1.6 \mathrm{C}$. As the results show that the $1.5 \mathrm{C}$ setting can give better results, this paper will include $1.5 \mathrm{C}$ as the normative threshold.

Figures 11-25 of this paper refers to the average of three games, not the result of a single game. The test process in the game is to automatically run the game in demo mode, 


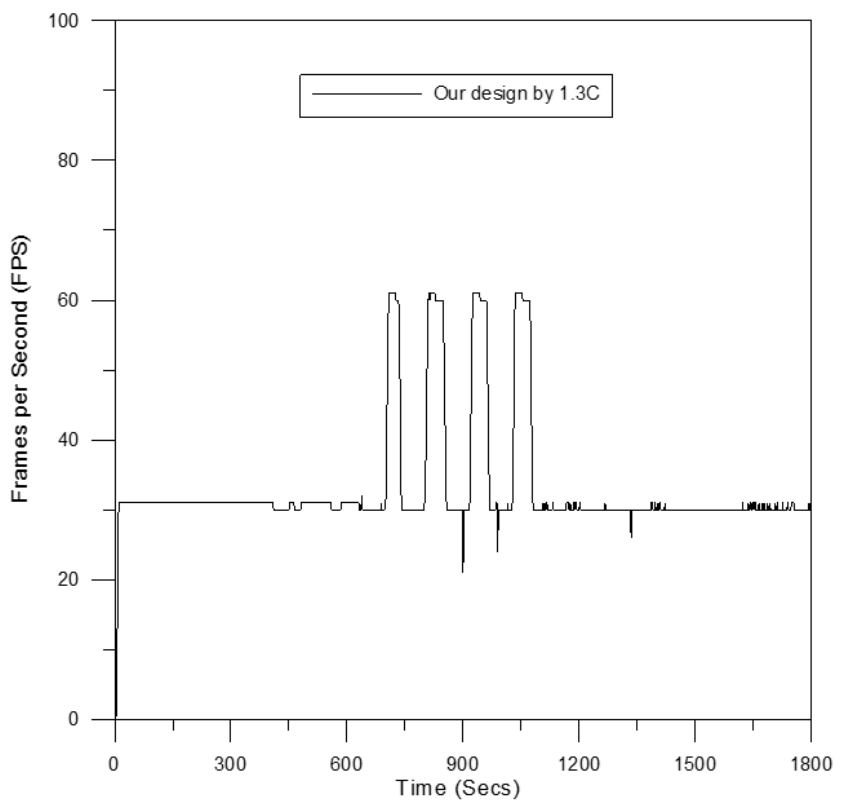

Fig. 24 The FPS with a 1.3C of our gaming notebook design.

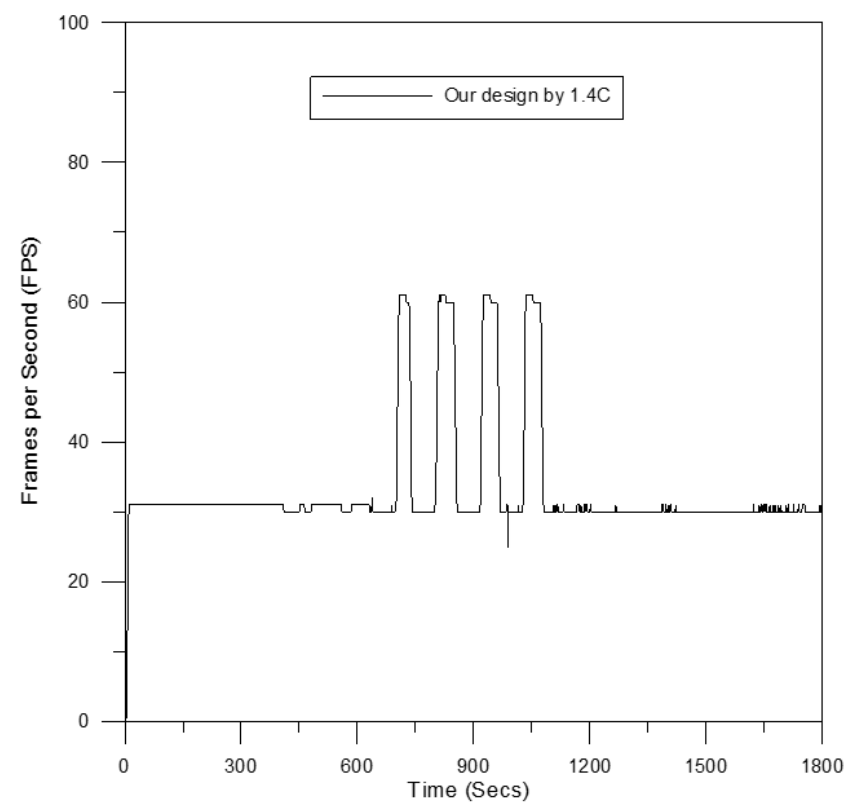

Fig. 25 The FPS with a 1.4C of our gaming notebook design.

Table 8 A comparison less than $30 \mathrm{FPS}$ of the $1.2 \mathrm{C}$ to $1.5 \mathrm{C}$.

\begin{tabular}{ccccc}
\hline & $1.5 \mathrm{C}$ & $1.4 \mathrm{C}$ & $1.3 \mathrm{C}$ & $1.2 \mathrm{C}$ \\
\hline Less than 30FPS & 0 & 1 & 3 & 5 \\
\hline
\end{tabular}

to record all data after running Demo mode, and finally to count the average of the three games.

The relevant settings in the game are compared to the default settings of the game itself. We did not specifically adjust the game.

Table 8 shows the comparison table of less than 30 FPS from $1.2 \mathrm{C}$ to $1.5 \mathrm{C}$ design. The number in the bottom row of
Table 9 The power consumption of the major modules of a typical gamming notebook.

\begin{tabular}{cc}
\hline & Power consumption \\
\hline CPU & $95 \mathrm{~W}$ (Maximum) \\
\hline GPU & $150 \mathrm{~W}$ (Maximum) \\
\hline Chipset & $15 \mathrm{~W}$ \\
\hline LCD Panel & 10W (Maximum) \\
\hline Other devices & 30W \\
\hline Total &
\end{tabular}

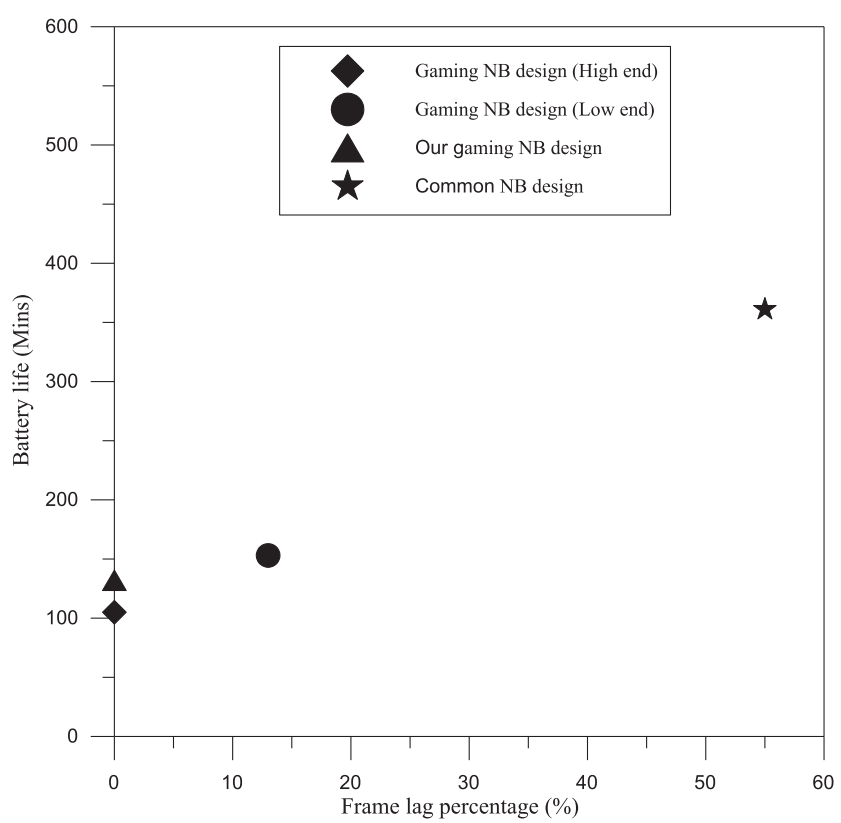

Fig. 26 "Battery life" vs. "Frame lag percentage".

Table 8 is "The number of frame lags".

Through experiments, we recorded the results of the discharge $\mathrm{C}$ number of $1.2 \mathrm{C}, 1.3 \mathrm{C}, 1.4 \mathrm{C}, 1.5 \mathrm{C}$, and finally found that the "Threshold" set to $1.5 \mathrm{C}$ is the best performance. Therefore, the "Threshold" of this paper is set to $1.5 \mathrm{C}$.

Table 9 shows the power consumption of the major modules of a typical gamming notebook. This design mainly emphasizes the power consumption of the CPU and the GPU. Although the complete system is not only the CPU and the GPU, but also the power consumption of other devices, the CPU and the GPU have already occupied about all of the overall available power according to the power consumption ratio. The CPU and the GPU consume $80-82 \%$, so our design is focused on the CPU and the GPU control.

We have measured the battery life of four different types of notebooks with respect to the performance and then provided a comparison to verify our design. This design has a better battery life and performance as shown in Fig. 26.

\section{4. $\quad$ Related Works}

Many GPUs have recently been used to accelerate the processing speed of the graphic data, and their applications provide simpler programmability and increased versatil- 
ity while simultaneously maintaining great memory bandwidth and computing power. Most of these applications use the CPU as a decision controller when the GPU runs computation-intensive tasks. This CPU-control-GPU computing mode wastes a lot of CPU power.

There are many different approaches to the study of CPU/GPU balance adjustments. Some research labs use compiler optimization methods for general CPUs [25], MPI [26], [27] to improve performance, and SIMD (Single Instruction Multiple Data) extended instructions for multiple data processing [28]. There are also some developments based on CuHMMer HMMER, which can increase the thread speed up by 13 to 45 times [29].

\section{Conclusion}

To balance the performance and the power consumption of the gaming notebook when used in the battery mode, we propose a new approach by using an EC with the BIOS with each other to control the CPU and GPU clock frequency. As long as the maximum discharge "C" number remains within a reasonable range in the battery mode of the gaming notebook, a high resolution video game still can be maintained at 30 FPS, so that users will not be affected by the frame lag phenomenon. Based on the experimental data, this design, with the dynamic adjustment both of the CPU and of the GPU clock frequency, is feasible, and will show a significantly better performance improvement than that of the common notebook when used in the battery mode. In comparison with the high end gaming notebook, this gaming notebook design has a better battery life with the same frame lag percentage which is $0 \%$. This design can provide users a sufficiently high speed and high resolution video game, such as the " $4 \mathrm{~K}$ " video game.

Based on the maximum discharge " $C$ " number, this design provides the necessary flexibility to dynamically adjust the CPU and the GPU clock frequency and then subsequently provide a complementary balance of the power consumption of the gaming notebook when used in the battery mode. By using this design, the battery life can be extended by about 27 minutes in comparison with that of a high end gaming notebook. For the gaming notebook market, this design can provide a " $4 \mathrm{~K}$ " video game with an around $0 \%$ frame lag percentage and a longer operation time in the battery mode. The purpose of achieving this quick control design is mainly because only the cooperation of the EC/BIOS can allow the gaming notebook to maintain a high performance in the battery mode, without the need for complicated hardware circuits or complicated algorithms. Instead, simple and flexible realization is the main purpose of this design. This design focuses the communication mechanism of the firmware program to control the EC/BIOS.

The purpose of this paper compares the four different systems, and as such, our comparison emphasizes the advantages of this specific design. Our design has advantages over other notebooks on the market today. Our design can maintain an excellent performance when in battery mode, which is not a design found in general notebook computers.

\section{Acknowledgments}

The authors gratefully acknowledge the technical support of the CLEVO Inc. for their suggestions and their review feedback about the notebook system included in this paper.

\section{References}

[1] C.-H. Cheng and Y.-W. Bai, "Anti-Ghost Key Design for a Notebook Keyboard," The 28th Annual IEEE Canadian Conference on Electrical and Computer Engineering (CCECE'2015), Halifax, Nova Scotia, Canada, pp.577-580, May 3-6, 2015.

[2] H. Kang, J. Park, and H. Bahn, "LBM: a low-power buffer management policy for heterogeneous storage in mobile consumer devices," IEEE Trans. Consum. Electron., vol.56, no.4, pp.2387-2392, Nov. 2010.

[3] D. Rakhmatov, S. Vrudhula, and D.A. Wallach, "A model for battery lifetime analysis for organizing applications on a pocket computer," IEEE Transactions on Very Large Scale Integration (VLSI) System, vol.11, no.6, pp.1019-1030, Dec. 2003.

[4] N. Vichare, P. Rodgers, V. Eveloy, and M.G. Pecht, "In situ temperature measurement of a notebook computer - a case study in health and usage monitoring of electronics," IEEE Trans. Device Mater. Rel., vol.4, no.4, pp.658-663, Dec. 2004.

[5] C.M. Olsen and C. Narayanaswarni, "PowerNap: an efficient power management scheme for mobile devices," IEEE Trans. Mobile Comput., vol.5, no.7, pp.816-828, July 2006.

[6] S. Thakkar, "Battery life challenges on future mobile notebook platform," Proc. 2004 International Symposium on Low Power Electronics and Design, ISLPED '04, p.187, Aug. 2004.

[7] A. Portero, G. Talavera, M. Moreno, J. Carrabina, and F. Catthoor, "Methodology for Energy-Flexibility Space Exploration and Mapping of Multimedia Application to Single-Processor Platform Styles," IEEE Trans. Circuits Syst. Video Technol., vol.21, no.8, pp.1027-1039, Aug. 2011.

[8] P. Yao, H. An, M. Xu, G. Liu, X. Li, Y. Wang, and W. Han, "A loadbalanced CPU-GPU cooperative bioinformatics application," IEEE 2010 International Conference on High Performance Computing and Simulation (HPCS), pp.24-30, 2010.

[9] L. Sant'Ana, D. Cordeiro, and R. Camargo, "PLB-HeC: A Profile-Based Load-Balancing Algorithm for Heterogeneous CPU-GPU Clusters," 2015 IEEE International Conference on Cluster Computing, pp.96-105, 2015.

[10] Y.-W. Bai and C.-H. Cheng, "Dynamic adjustment of processor clock frequency to prevent notebook from shutdown by AC adapter due to overheating," The 1st IEEE Global Conference on Consumer Electronics, Tokyo, Japan, pp.520-524, Oct. 2-5, 2012.

[11] M.-H. Cho and C.-H. Lee, "A low-power real-time operating system for ARC(actual remote control) wearable device," IEEE Trans. Consum. Electron., vol.56, no 3, pp.1602-1609, Aug. 2010.

[12] A. Stroppa, C. Spini, and C. Adragna, "High performance AC-DC notebook PC adapter meets EPA 4 requirements," 13th European Conference on Power Electronics and Applications EPE '09, pp.19, 2009.

[13] J. Charles, P. Jassi, N.S. Ananth, A. Sadat, and A. Fedorova, "Evaluation of the Intel Core i7 Turbo Boost Feature," International Symposium on Workload Characterization IISWC 2009, pp.188-197, 2009.

[14] C.-H. Cheng and Y.-W. Bai, "Using Fuzzy Logic and Light-Sensor for Automatic Adjustment of Backlight Brightness in a Mobile Computer," The 16th IEEE International Symposium on Consumer Electronics, Harrisburg, Pennsylvania, USA, pp.47-51, June 4-6, 2012.

[15] C.-H. Cheng and Y.-W. Bai, "A Peak-Shift Control Method for 
Charging and Discharging of the Battery in an Ultrabook," IEEE International Conference on Consumer Electronics, pp.296-297, Jan. 2014.

[16] Y.-W. Bai and C.-H. Cheng, "Using Fuzzy Logic to Reduce Power Consumption for Notebooks," IEEE The 13th International Symposium on Consumer Electronics, pp.488-492, May 2009.

[17] Y.-W. Bai and C.-H. Cheng, "Using an embedded controller with fuzzy logic to reduce power consumption of mobile computers," The 36th Annual Conference of the IEEE Industrial Electronics Society (IECON-2010), Phoenix, Arizona, USA, pp.230-235, Nov. 7-10, 2010.

[18] C.-H. Cheng and Y.-W. Bai, "An Automatically Peak-Shift Control Design for Charging and Discharging of the Battery in an Ultrabook," IEICE Trans. Inf. \& Syst., vol.E99-D, no.4, pp.1108-1116, April 2016.

[19] R. Shea, D. Fu, and J. Liu, "Cloud Gaming: Understanding the Support From Advanced Virtualization and Hardware," IEEE Transaction on Circuits and System for Video Technology, vol.25, no.12, pp.2026-2037, Dec. 2015

[20] T. Komoda, S. Hayashi, T. Nakada, S. Miwa, and H. Nakamura, "Power capping of CPU-GPU heterogeneous systems through coordinating DVFS and taskmapping," 2013 IEEE 31st International Conference on computer design(ICCD), pp.349-356, Oct. 2013.

[21] A. Pathania, Q. Jiao, A. Prakash, and T. Mitra, "Integrated CPU-GPU power management for 3D mobile games," Proc. 51st Annual Design Automation Conference, DAC '14, pp.1-6, June 2014.

[22] J.-G. Park, C.-Y. Hsieh, N. Dutt, and S.-S. Lim, "Co-Cap: energy-efficient cooperative CPU-GPU frequency capping for mobile games," Proc. 31st Annual ACM Symposium on Applied Computing, SAC '16, pp.1717-1723, April 2016.

[23] http://www.trendforce.com

[24] http://www.digitimes.com.tw

[25] J. Landman, J. Ray, and J.P. Walters, "Accelerating HMMer searches on Opteron processors with minimally invasive recoding," Proc. HiPCOMB 2006, Vienna, Austria, 2006.

[26] J.P. Walters, B. Qudah, and V. Chaudhary, "Accelerating the HMMER Sequence Analysis Suite Using Conventional Processors," AINA 2006, Vienna, Austria, 2006.

[27] J.P. Walters, R. Darole, and V. Chaudhary, "Improving MPI-HMMER's scalability with parallel I/O," Proc. 2009 IEEE International Symposium on Parallel \& Distributed Processing, IPDPS 2009, May 2009.

[28] E. Lindahl, Altivec HMMer, version 2, 2009. [Online] Available: http://lindahl.sbc.su.se/software/altivec/altivechmmer-version2.html.

[29] P. Yao, H. An, M. Xu, G. Liu, X. Li, Y. Wang, and W. Han, "CuHMMer: A load-balanced CPU-GPU cooperative bioinformatics application," IEEE 2010 International Conference on High Performance Computing and Simulation (HPCS), pp.24-30, 2010.

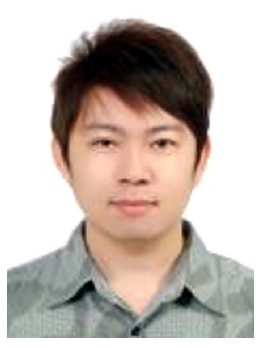

Chun-Hung Cheng is currently working toward the Ph.D. degree in Graduate Institute of Applied Science and Engineering at Fu Jen Catholic University, Taiwan. His research interests include low power system design, mobile computing, and embedded computer systems. He holds a post in CLEVO Company and takes on the position of software design department of research and development centre at present.

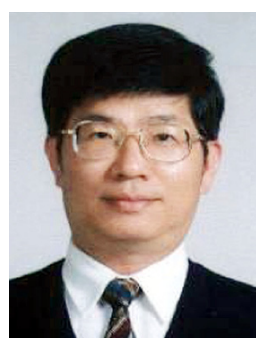

Ying-Wen Bai is a professor in the Department of Electrical Engineering at Fu Jen Catholic University. His research focuses on mobile computing and microcomputer system design. He obtained his M.S. and Ph.D. degrees in electrical engineering from Columbia University, New York, in 1991 and 1993, respectively. Between 1993 and 1995, he worked at the Institute for Information Industry, Taiwan. 